\title{
Selective Alveolo-bronchographic Findings in Type B Chronic Obstructive Lung Disease Patients with Particular Reference to Lesions in Small Airways
}

\author{
Shigeru Sato, Masahiko Kawakami, Sanae Shimura, \\ Ryuitsu Fujimoto, Toshio Hrrose, Shinsaku Maeda, \\ Tadashi Ubukata and Tamotsu Takishima \\ The First Department of Internal Medicine, Tohoku University \\ School of Medicine, Sendai 980
}

\begin{abstract}
Sato, S., Kawakami, M., Shmmora, S., Fujmoto, R., Hrrose, T., Maeda, S., Ubukata, T. and Takishima, T. Selective Alveolo-bronchographic Findings in Type $B$ Chronic Obstructive Lung Disease Patients with Particular Reference to Lesions in Small Airways. Tohoku J. exp. Med., 1977, 123 (2), 99-108Selective alveolo-bronchography (SAB) was performed in 14 patients with type B chronic obstructive lung disease to evaluate the morphologic changes of their "small airways". The distance from the branching portion of the segmental bronchus $\left(B_{9}\right)$ to the end of the conducting airway was divided into three equal parts (central, middle and peripheral). In the peripheral part, interruption of the airway with a rounded or tapering end was noted at a high incidence $(9$ cases). Dilatation of the bronchus, irregular outline of the bronchial wall and increased angle of branching were also conspicuous in this part. Inadequate lateral branchings of airways were often observed in the middle and the peripheral part, particularly in the latter (10 cases). Generalized narrowing of an airway and narrowing especially at a branching point were observed in about one half of the cases. Fringes and/or spikes were most often seen in the central part of the airway (9 cases). These pathological appearances are considered to reflect morphologic changes in "small airway disease", and thus SAB can serve as a useful method for detecting lesions of the small airways. _—_ selective alveolo-bronchography; type $\mathrm{B}$ chronic obstructive lung disease; small airways
\end{abstract}

It was experimentally demonstrated by Macklem and Mead (1967) that the resistance afforded by airways less than $2 \mathrm{~mm}$ in diameter contributed little to the total pulmonary resistance and that considerable obstruction might occur at these sites with little effect on the overall resistance. Later, Hogg et al. (1968) showed in excised human lungs that diseases of small airways were common to various chronic obstructive lung disease (COLD) states, and that the obstruction in COLD occurred in airways smaller than $2 \mathrm{~mm}$ in diameter. It is now believed that the respiratory insufficiency in chronic bronchitis, bronchiectasis and probably most of the cases of pulmonary emphysema may be attributable to the obstruction of small airways.

Thus, changes in small airways have come to attract much attention in recent

Received for publication, April 8, 1977. 
years. However, as mentioned above, it is difficult to detect obstruction of the small airways by physiological examinations. In 1969, Nakamura et al. developed selective alveolo-bronchography (SAB), a radiographic procedure whereby destruction of the parenchyma of the lung is detected at the morphologic level in patients with chronic pulmonary emphysema. In considering that this technique might be also a useful radiographic procedure in evaluating diseases of small airways, we have been conducting studies on the pathological appearances of small airways in patients with COLD using this method.

In type B COLD patients, the emphysematous change is either absent or mild. It is considered that the respiratory insufficiency in this type is caused by changes in airways $2 \mathrm{~mm}$ or less in diameter. Therefore, type B patients with COLD strongly suspected of having small airway obstructions were examined by $\mathrm{SAB}$. The purpose of the present study is largely to define pathological appearances of the small airways that cannot be adequately revealed by plain radiography or ordinary bronchography.

\section{Materials and Methods}

The method of selective alveolo-bronchography (SAB) has been described in detail elsewhere (Nakamura et al. 1969). In essence, a special catheter is inserted selectively into a single bronchus of the right or left lower lobe (usually $B_{9}$ ), its tip prewedged in the subsegmental bronchus. 5 to $8 \mathrm{ml}$ of the contrast medium (aqueous Dionosil) are gradually introduced by a syringe via one of the polyethylene tubes fixed inside the catheter, and immediately after the contrast medium appears at the tip of the catheter, air is introduced by a compressor via the other polyethylene tube fixed inside the catheter. The pulmonary region filled with the contrast medium is visualized under a magnification of three times.

In interpreting $\mathrm{SAB}$ films, the airway visualized was divided into three equal parts over the distance from the branching portion of the segmental bronchus down to the end of the airway. They were called the central part (A), the middle part (B) and the peripheral part (C), respectively (Fig. 1). The studies were made on the presence or absence and the degree of pathological findings in each division.

Using this technic, lungs from 14 cases were studied. They had an irreversible obstructive impairment with FEVI/FVC lower than $70 \%$ and showed at least three of the following characteristics proposed by Burrows et al. (1966) but no radiographic evidence of emphysema: (1) persistent expectoration of sputum more than $10 \mathrm{ml} /$ day; (2) disproportionate hypercapnia $\left(\mathrm{Pa}_{\mathrm{CO}_{2}}\right) 6 \mathrm{mmHg}$ or more above the level predicted from the $\left.\mathrm{FEVI}\right)$; (3) definite $X$-ray evidence of chronic inflammatory disease in the lung parenchyma; (4) TLC less than $100 \%$ of the predicted value; (5) well-preserved diffusing capacity $\left(D_{L} / V_{A} 3.5\right.$ or more); and (6) chronic or recurrent heart-failure due to persistent cor pulmonale.

\section{Results}

In Tables 1 and 2 are shown the clinical data and results of pulmonary function tests on 14 patients.

A selective alveolo-bronchogram of a healthy male is presented in Fig. 1. The alveolar pattern was revealed as collections of very small dots. Both the bronchus and the bronchioles exhibited the normal pattern of branching. In Table 3 is shown the frequency of pathological appearances in each division of the 
Table 1. Clinical data on 14 patients

\begin{tabular}{|c|c|c|c|c|c|c|c|c|c|}
\hline \multicolumn{3}{|c|}{ Case } & \multirow{2}{*}{$\begin{array}{c}\text { Age } \\
\text { at } \\
\text { onset }\end{array}$} & \multirow{2}{*}{ Initial complaints } & \multirow{2}{*}{$\begin{array}{l}\text { Smoking* } \\
\text { history }\end{array}$} & \multirow{2}{*}{ Sputum $†$} & \multirow{2}{*}{ Dyspnea +} & \multirow{2}{*}{$\begin{array}{c}\text { Heart } \\
\text { failure§ }\end{array}$} & \multirow{2}{*}{$\underset{\left(\times 10^{4}\right)}{\mathrm{RBC} / \mathrm{mm}^{3}}$} \\
\hline No. & Age & Sex & & & & & & & \\
\hline 1 & 30 & M & 15 & Cough \& sputum & $\operatorname{sm}$ & + & 1 & & 550 \\
\hline 2 & 59 & M & 44 & Dyspnea & non & + & 2 & & 556 \\
\hline 3 & 46 & F & 36 & Dyspnea & non & + & 2 & & 376 \\
\hline 4 & 75 & M & 30 & Cough \& sputum & $\mathrm{sm}$ & + & 3 & + & 581 \\
\hline 5 & 44 & M & 20 & Cough \& sputum & ex & H & 4 & & 547 \\
\hline 6 & 63 & M & 39 & Cough \& sputum & ex & $H$ & 2 & & 329 \\
\hline 7 & 44 & $\mathbf{F}$ & 36 & Cough \& sputum & non & + & 4 & & 469 \\
\hline 8 & 56 & $\mathrm{M}$ & 20 & Sputum & $\mathrm{sm}$ & $\mathrm{Ht}$ & 3 & & $?$ \\
\hline 9 & 72 & M & 50 & Stridor & non & + & 4 & + & 505 \\
\hline 10 & 55 & M & 44 & Dyspnea & non & 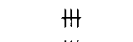 & 4 & & 534 \\
\hline 11 & 47 & M & 37 & Cough & non & $H$ & 3 & & 532 \\
\hline 12 & 42 & M & 39 & Dyspnea & ex & + & 2 & & 520 \\
\hline 13 & 45 & M & 13 & Cough & non & $H$ & 4 & + & 398 \\
\hline 14 & 39 & M & 32 & Stridor & non & $H$ & 4 & & 512 \\
\hline
\end{tabular}

* ex: exsmoker, sm: smoker, non: nonsmoker.

$++: 50 \mathrm{ml}$ or less per day, + : 50 to $160 \mathrm{ml}$ per day, $\mathrm{H}: 100 \mathrm{ml}$ or more per day.

+ Hugh-Jones' classification.

$\$+$ : Chronic or recurrent right heart failure due to chronic cor pulmonale.

TABLE 2. Results of pulmonary function tests in individual patients

\begin{tabular}{|c|c|c|c|c|c|c|}
\hline Case No. & $\begin{array}{c}\text { VC } \\
\text { (o pred.) }\end{array}$ & $\mathrm{FEV}_{1} / \mathrm{FVC}, \%$ & $\mathrm{DLCO} / V_{\mathrm{A}}$ & $\begin{array}{c}\text { TLC } \\
\text { (\% pred.) }\end{array}$ & $\begin{array}{c}\mathrm{PaO}_{2} \\
(\mathrm{mmHg})\end{array}$ & $\underset{(\mathrm{mmHg})}{\mathrm{PaCO}_{2}}$ \\
\hline 1 & -3 & 68.7 & 6.10 & +11 & 72.0 & 35.0 \\
\hline 2 & -19 & 31.0 & 8.29 & +11 & 60.0 & 35.0 \\
\hline 3 & -34 & 54.5 & 7.40 & -8 & 66.0 & 33.5 \\
\hline 4 & -35 & 56.5 & 4.79 & -1 & 60.0 & 47.0 \\
\hline 5 & -43 & 28.0 & 7.00 & +13 & 55.0 & 57.0 \\
\hline 6 & +3 & 42.2 & 3.70 & -19 & 73.7 & 35.5 \\
\hline 7 & 0 & 42.0 & 6.45 & +24 & 61.0 & 40.0 \\
\hline 8 & -52 & 50.8 & 5.90 & -29 & 68.0 & 37.0 \\
\hline 9 & -27 & 42.5 & 2. 30 & +3 & 50.8 & 49.5 \\
\hline 10 & -20 & 32.6 & 5. 31 & -13 & 58.0 & 41.8 \\
\hline 11 & -37 & 47.3 & 6.00 & -23 & 74.8 & 43.2 \\
\hline 12 & -54 & 31.0 & 6.70 & -19 & 59.4 & 40.7 \\
\hline 13 & -44 & 48.0 & 6.00 & -13 & 59.4 & 49.0 \\
\hline 14 & -55 & 41.0 & 6.60 & -26 & 52.0 & 35.2 \\
\hline
\end{tabular}

airway and the alveolar region.

Peripheral part of the airway. General findings characteristic of this part were peripheral non-filling of the contrast medium (the interrupted pattern). The airways were interrupted with a tip describing a semi-circle (Fig. 2) (6 cases, 43\%), or with a dilated or tapered end (Fig. 3) (6 cases, 43\%). In most instances, the interrupted patterns were distributed sporadically, but in 2 cases all of the visualized airways showed rounded ends (Fig. 4).

Dilatation of the airways was observed in 13 cases, $93 \%$ (cylindrical, 10 cases; saccular, 4 cases). Irregular outlines of the bronchial wall (Fig. 5) were also noted at a high incidence in 12 cases, $86 \%$ (concertina-like appearance, 10 cases; beaded 


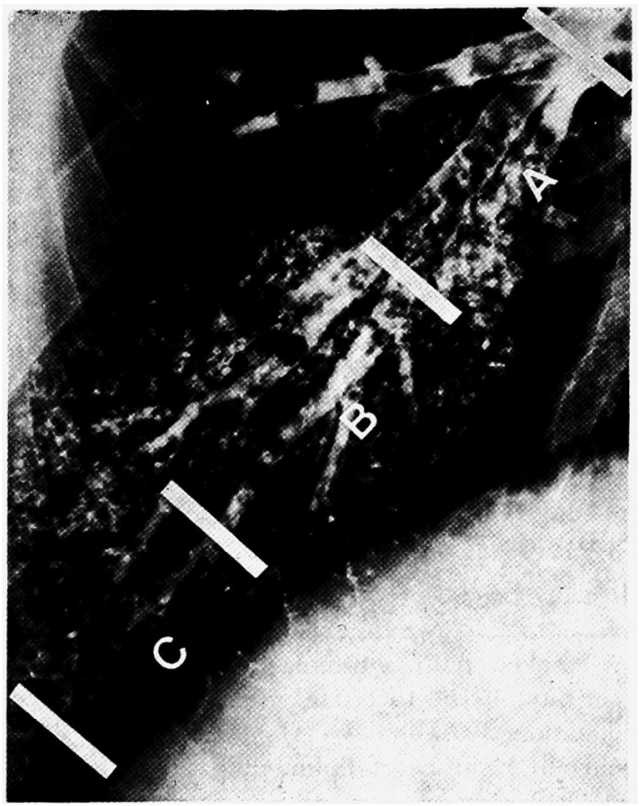

Fig. 1. A selective alveolo-bronchogram of human lung (a 53 year-old male). The distance from the branching portion of the segmental bronchus down to the end of the conducting airway is routinely divided into three equal parts; the central part (A), the middle part (B) and the peripheral part (C). The alveolar pattern is normal and both the bronchus and the bronchioles exhibit the normal pattern of branching.

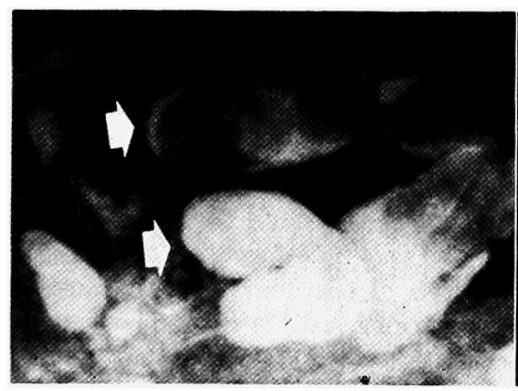

Fig. 2

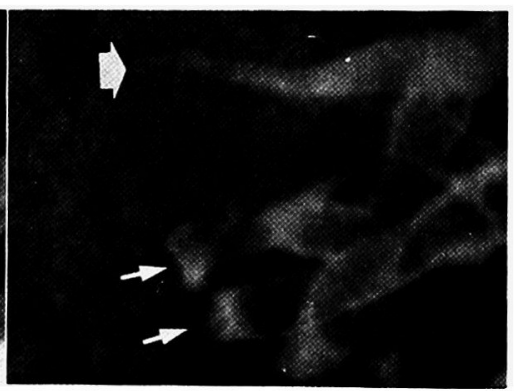

Fig. 3

Fig. 2 (left). Area of the lung showing rounded endings with cylindrical dilatation (arrows). The peripheral part of the airway. Case 13.

Fig. 3 (right). Area of the lung showing tapering endings (large arrow) and rectilinear endings (small arrows). The peripheral part of the airway. Case 9.

appearance, 4 cases; others, 8 cases). Furthermore, there was observed inadequate lateral branching of airways (Fig. 6) in 10 cases, $71 \%$; airway narrowing in 8 cases, $57 \%$ (of these generalized narrowing, 7 cases; narrowing at a branching point, 3 cases); and an increased angle of branching - where the branching angle of the airways was increased to 90 degree or more - in 5 cases, $36 \%$. 
TABLE 3. Frequency of selective alveolo-bronchographic findings at each part of the airway and in the alveolar region in 14 patients

\begin{tabular}{lccc}
\hline \multirow{2}{*}{ Findings of airways } & \multicolumn{3}{c}{ Numbers of cases } \\
\cline { 2 - 4 } & Peripheral part & Middle part & Central part \\
\hline Fringe and/or spike & $1(7 \%)$ & $1(7 \%)$ & $9(64 \%)$ \\
Irregular contour & $12(86 \%)$ & $11(79 \%)$ & $7(50 \%)$ \\
Concertina-like appearance & 10 & 7 & 0 \\
Beaded appearance & 4 & 3 & 0 \\
Others & 8 & 8 & 7 \\
Dilatation & $13(93 \%)$ & $11(79 \%)$ & $5(36 \%)$ \\
Cylindrical & 10 & 8 & 5 \\
Saccular & 4 & 3 & 1 \\
Loss of taper & 8 & 5 & $6(43 \%)$ \\
Narrowing & $8(57 \%)$ & $8(57 \%)$ & 4 \\
Generalized & 7 & 4 & 3 \\
At a branching point & 3 & 5 & $0(0 \%)$ \\
Inadequate lateral branching & $10(71 \%)$ & $8(57 \%)$ & $0(0 \%)$ \\
of airways & & & $0(0 \%)$ \\
Increased angle of branching & $5(36 \%)$ & $0(0 \%)$ & 0 \\
Ending & $9(6496)$ & $1(7 \%)$ & 0 \\
Rounded & 6 & 1 & 0 \\
Tapering & 6 & 0 & \\
Rectilinear & 4 & &
\end{tabular}

Findings in the alveolar region Numbers of cases

\begin{tabular}{lr} 
Alveolar filling & \\
Normal & 2 \\
Uneven & 4 \\
Poor or absent & 10 \\
Alveolar pattern & \\
Normal & 4 \\
Coarse & 3 \\
Destructive & 0 \\
\hline
\end{tabular}

Middle part of the airway. This part of the airway showed marked changes, particularly at a portion adjacent to the peripheral part. Irregularity of the wall was noted in 11 cases, $79 \%$ (of these concertina-like appearance, 7 cases; beaded appearance, 3 cases; others, 8 cases); dilatation of the airway also occurred frequently in 11 cases, $79 \%$ (of these cylindrical, 8 cases; saccular, 3 cases; loss of taper, 5 cases). In addition, significant airway narrowing was observed in 8 cases, $57 \%$ (generalized narrowing, 4 cases; narrowing at a branching point, 5 cases) and inadequate lateral branching of airways in 8 cases, $57 \%$.

Central part of the airway. Changes in this part were mild as compared with the other parts. That is, fringes and/or spikes (Fig. 7) were seen at the highest rate in 9 cases, $64 \%$; followed by irregularity of the wall in 7 cases, $50 \%$; dilatation in 5 cases, $36 \%$ (cylindrical, 5 cases; loss of taper, 1 case); and airway narrowing (Fig. 8) in 6 cases, $43 \%$ (generalized narrowing, 6 cases; narrowing at a branching 


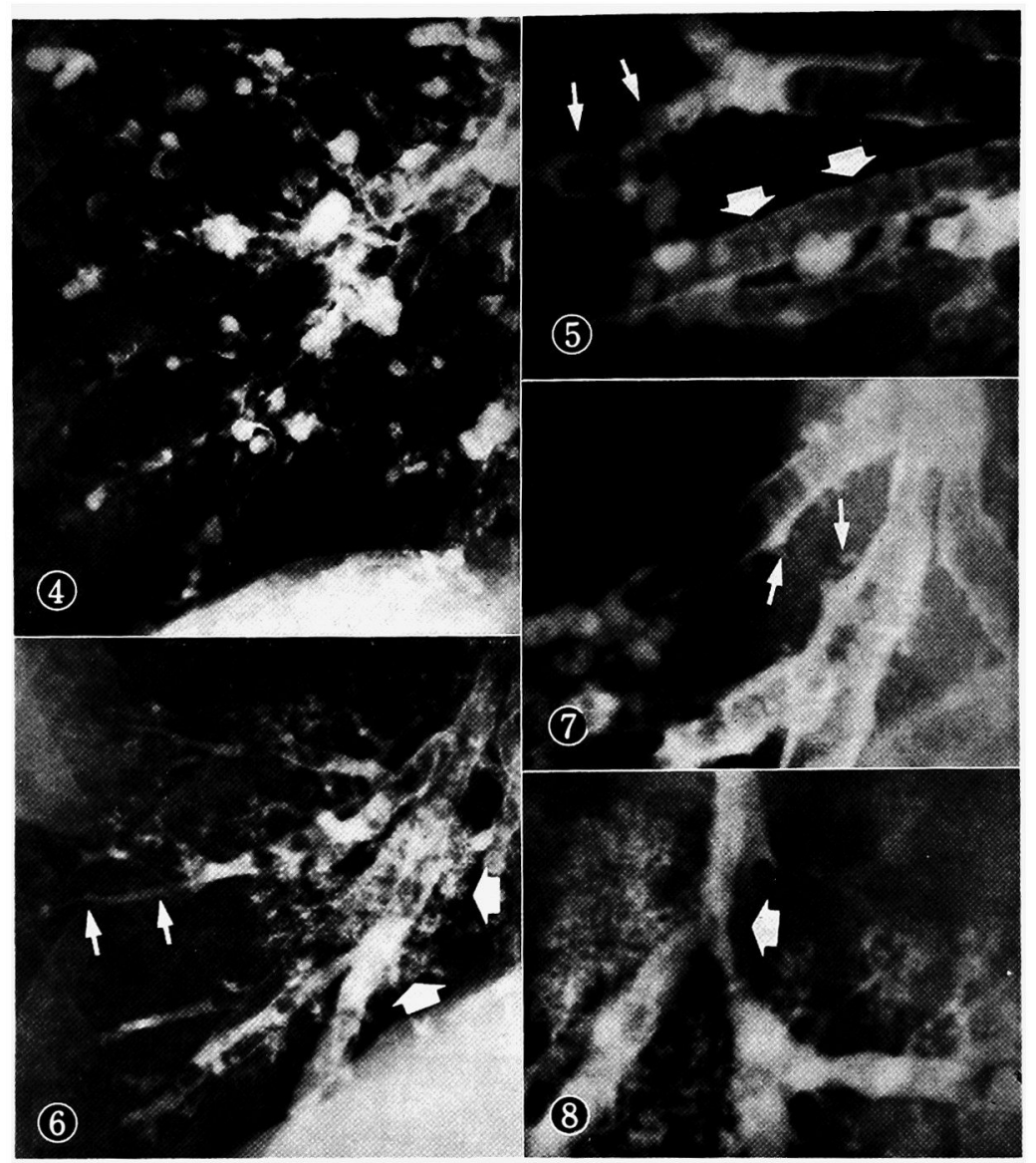

Fig. 4. A selective alveolo-bronchogram Iof Case 14. All airways show rounded endings at the peripheral part.

Fig. 5. Area of the lung showing concertina-like appearance (large arrow) and beaded appearance (small arrow). The peripheral part of the airway. Case 5 .

Fig. 6. A selective alveolo-bronchogram of Case 7. Note inadequate lateral branching of airways (small arrows) and uneven alveolar filling (large arrow).

Fig. 7. A selective alveolo-bronchogram of the human lung showing fringes and spikes (arrows). The central part of the airway. Case 9.

Fig. 8. Area of the lung showing narrowing at a branching point. The central part of the airway. Case 4.

point, 3 cases).

Alveolar region. The alveolar pattern was seldom visualized clearly. 12 cases, $86 \%$ showed abnormalities of various forms in appearance of the alveolar pattern. 10 cases, $71 \%$ showed poor or no filling of the contrast medium. 4 cases, $29 \%$ showed uneven filling (Fig. 6), that is, the coexistence of excessively instilled portions and no filling portions on one sheet of film. In the case of poor or excessive filling, it was not easy to judge the alveolar structure from SAB. However, the judgement could be made in 7 cases; none showed the destruction of 
alveolar structure suspected of pulmonary emphysema, and a roughened pattern was noted only in 3 of these cases. In 4 cases, the alveolar structure was judged to be normal.

In Table 4 are shown the degrees of pathological findings in SAB from the peripheral part and the alveolar region in individual patients. The degrees are expressed in terms of the length of airway affected; when the changes cover $1 / 3$ or less of the airway they are rated as one-plus $(+)$, those of $1 / 3-2 / 3$ as two-pluses $(H)$, and more than $2 / 3$ as three-pluses $(H)$.

All 14 cases showed some pathological findings, though their degrees were not the same. Changes were mild in Cases 1-4, and varied and extensive in the other cases. All cases except Cases 3 and 4 showed abnormalities of the alveolar pattern.

SAB findings from the middle and central part are summarized in Table 5 . All cases showed pathological appearances of various forms as in the peripheral part. Whereas changes were slight in Cases $1-4$, they were multifarious and extensive in the remaining cases. Irregularities of the wall and cylindrical dilatations were conspicuous in Cases 6-14.

\section{Discusston}

All cases of type B chronic obstructive lung disease submitted to the present study had histories of recurrent infection of the bronchial airways with moderate to severe obstructive impairments and the production of large amounts of purulent sputum over many years. Relatively young persons whose age at the onset of illness was below 40 years accounted for the majority of the present cases. Among them, those of greater severity showed marked shortness of breath, hypoxemia and polycythemia, and were often accompanied by heart failure.

The presence of a fringe or spike was observed in 9 out of 14 cases $(64 \%)$. This high rate was probably due to the fact that the subjects examined consisted of those who had a long-standing history of excess sputum and also that minute changes had been visualized clearly under a magnification of three times.

The interrupted pattern of the peripheral airway has not so far been paid much attention. Reid (1955) made it clear, by correlated bronchographical and histopathological studies of excised lungs in 11 cases of chronic bronchitis, that the rounded ending and the tapered ending in bronchography suggest organic occlusions, and that the rectilinear ending can be caused by a failure in instillation of the contrast medium further down to the bronchial tree. That the peripheral part of the airway was hard to visualize in this disease was also pointed out by Simon and Galbraith (1953) and was considered to be due to poor filling of smaller bronchi and bronchioli.

Burrows et al. (1966) pointed out that the emphysematous changes were mild histopathologically in cases of type B COLD compared with those of type A, and that the main part of lesion of type $B$ would be located in the airway. The present study revealed that although changes were most conspicuous in the 
TABLE 4. Selective alveolo-bronchographic findings at the peripheral part

\begin{tabular}{|c|c|c|c|c|c|c|}
\hline \multirow{2}{*}{$\begin{array}{l}\text { Case } \\
\text { No. }\end{array}$} & \multicolumn{2}{|c|}{ Ending* } & \multirow{2}{*}{$\begin{array}{c}\text { Inadequate } \\
\text { lateral branching } \\
\text { of airways* }\end{array}$} & \multicolumn{3}{|c|}{ Irregular contour $\uparrow$} \\
\hline & Rounded & Tapering & & $\begin{array}{l}\text { Concertina-like } \\
\text { appearance }\end{array}$ & $\begin{array}{c}\text { Beaded } \\
\text { appearance }\end{array}$ & Others \\
\hline 1 & & & & & & + \\
\hline 2 & & & & & & \\
\hline 3 & & & & & & \\
\hline $\begin{array}{l}4 \\
5\end{array}$ & & & $t$ & + & + & $H$ \\
\hline 5 & & + & $\begin{array}{l}+ \\
+\end{array}$ & $\begin{array}{l}+ \\
+\end{array}$ & + & H \\
\hline $\begin{array}{l}6 \\
7\end{array}$ & & + & + & + & & \\
\hline 8 & & + & + & + & & $\mathrm{HH}$ \\
\hline 9 & + & + & + & + & & + \\
\hline 10 & + & + & + & H & + & \\
\hline 11 & + & + & + & + & + & H \\
\hline 12 & + & & + & + & + & H \\
\hline 13 & all & & + & & & \\
\hline 14 & all & & + & + & & H \\
\hline
\end{tabular}

* $+:$ The presence of the findings is clear. all: All airways showed rounded endings.

$\dagger+:$ seen in less than $1 / 3$ of the extent, $H:$ seen in $1 / 3$ to $2 / 3$ of the extent, $H$ : seen in more than $2 / 3$ of the extent.

TABLE 5. Selective alveolo-bronchographic findings at the central

\begin{tabular}{|c|c|c|c|c|c|}
\hline \multirow[b]{2}{*}{ Case No. } & \multirow{2}{*}{$\begin{array}{l}\text { Spike and/or } \\
\text { fringe* }\end{array}$} & \multicolumn{3}{|c|}{ Irregular contour $\dagger$} & \multirow[b]{2}{*}{ Cylindrical } \\
\hline & & $\begin{array}{l}\text { Concertina-like } \\
\text { appearance }\end{array}$ & $\begin{array}{c}\text { Beaded } \\
\text { appearance }\end{array}$ & Others & \\
\hline 1 & + & & & & \\
\hline 2 & & & & & \\
\hline 3 & & & & & \\
\hline 4 & & + & + & & \\
\hline 5 & + & + & & + & \\
\hline 6 & & + & & \# & \# \\
\hline 7 & + & + & & + & H \\
\hline 8 & + & + & & $\mathrm{HH}$ & H \\
\hline 9 & + & & + & + & + \\
\hline 10 & + & H & & & \\
\hline 11 & + & & + & $\mathrm{HW}$ & + \\
\hline 12 & + & & & $\mathrm{HH}$ & \# \\
\hline 13 & + & & & + & $\mathrm{H}$ \\
\hline 14 & & + & & $\mathrm{H}$ & H \\
\hline
\end{tabular}

$*+:$ The presence of the findings is clear.

++ : seen in less than $1 / 3$ of the extent, $\#:$ seen in $1 / 3$ to $2 / 3$ of the extent, H: seen in more than $2 / 3$ of the extent.

middle and the peripheral part of the airway, not a single case showed definite emphysematous changes of the alveoli. In addition, the possibility cannot be ruled out that mild emphysematous changes could escape detection since the alveoli are difficult to visualize and sufficient information on changes in the alveolar region is hard to obtain in this disease.

When calculated from the morphological measurement of airways of the normal lung performed by Horsfield and Cumming (1968), the inside diameter of a 
of the airway and in the alveolar region in individual patients

\begin{tabular}{|c|c|c|c|c|c|c|}
\hline \multicolumn{3}{|c|}{ Dilatationt } & \multicolumn{2}{|c|}{ Narrowing } & \multicolumn{2}{|c|}{ Alveolar filling* } \\
\hline Cylindrical & Saccular & $\begin{array}{l}\text { Loss of } \\
\text { taper }\end{array}$ & Generalized $\dagger$ & At a branching point* & Uneven & $\begin{array}{l}\text { Poor or } \\
\text { absent }\end{array}$ \\
\hline & & + & & & & + \\
\hline & & + & & + & & + \\
\hline & & & \# & & & \\
\hline & & & + & + & & \\
\hline & & $H$ & & & + & \\
\hline+ & & + & H & & + & + \\
\hline+ & & & $H$ & & + & \\
\hline H & + & + & & & & + \\
\hline+ & + & + & H & & & + \\
\hline$H$ & & & + & + & & + \\
\hline+ & + & $\mathrm{Ht}$ & + & & & + \\
\hline+ & & $H$ & & & + & + \\
\hline+ & + & & & & & + \\
\hline HH & & & & & & + \\
\hline
\end{tabular}

and middle part of the airway in individual patients

\begin{tabular}{|c|c|c|c|c|}
\hline \multicolumn{2}{|l|}{ Dilatation $\uparrow$} & \multicolumn{2}{|c|}{ Narrowing } & \multirow{2}{*}{$\begin{array}{l}\text { Inadequate } \\
\text { lateral branching } \\
\text { of airways* }\end{array}$} \\
\hline Saccular & $\begin{array}{c}\text { Loss of } \\
\text { taper }\end{array}$ & Generalized $\dagger$ & At a branching point* & \\
\hline & + & & + & \\
\hline & + & \# & + & \\
\hline & & + & & \\
\hline & + & & + & + \\
\hline & + & & & $\begin{array}{l}t \\
t\end{array}$ \\
\hline \multirow{2}{*}{+} & & Ht & + & \\
\hline & & + & & + \\
\hline+ & + & & & + \\
\hline \multirow[t]{3}{*}{+} & & + & + & + \\
\hline & & & & + \\
\hline & & & + & + \\
\hline
\end{tabular}

part corresponding to the boundary between the middle and the peripheral part of the airway is approximately $2.4 \mathrm{~mm}$ in the left lung; whereas, when the data of Weibel (1963) are used, it is estimated to be approximately $1.5 \mathrm{~mm}$. Using the lungs of patients with various diseases, Hogg et al. (1968) measured the resistance of central and peripheral airways; the latter had diameters $2 \mathrm{~mm}$ or less. So, the peripheral resistance measured by them almost corresponded to the resistance of peripheral part of the present authors. Macklem et al. (1971) examined, clinically 
and pathologically, 7 patients with diffuse, reticular shadows on chest X-ray film, shortness of breath, cough and sputum. These patients were similar to ours in that most of them had restrictive impairment, obstructive impairment, polycythemia, respiratory insufficiency and cor pulmonale. Their pathological investigation revealed fibrosis around the bronchioli, inflammatory changes such as obstructive bronchitis, and mucous pluggings. In the present study, the authors were able to detect by $\mathrm{SAB}$ a high frequency of rounded endings, tapering endings, inadequate lateral branchings of airways and abnormalities in appearance of the alveolar pattern. These findings are considered to represent the small airway lesions that Macklem et al. (1971) observed.

Formerly, SAB was regarded as an in-vivo method for clinico-morphologic diagnosis of chronic pulmonary emphysema. In recent years, considerable interest has been evoked in the morphology of small airways in relation to "small airway disease". With ordinary bronchography, however, it is difficult to analyze small airway lesions. SAB has proved to be excellent in detecting delicate changes in small airways as well as in alveolar regions, and is now expected to be an effective radiographic procedure for detecting small airway disease.

The authors, for the present, have no results concerning the correlation between $\mathrm{SAB}$ and histopathological findings in small airways. In order to confirm the reliability of $\mathrm{SAB}$ findings, pathological investigations on clinical cases in which SAB has been carried out will be needed.

\section{References}

1) Burrows, B., Fletcher, C.M., Heard, B.E., Jones, N.L. \& Wootliff, J.S. (1966) The emphysematous and bronchial types of chronic airways obstruction. Lancet, 1, 830835 .

2) Hogg, J.C., Macklem, P.T. \& Thurlbeck, W.M. (1968) Site and nature of airway obstruction in chronic obstructive lung disease. New Engl. J. Med., 278, 1355-1360.

3) Horsfield, K. \& Cumming, G. (1968) Morphology of the bronchial tree in man. J. appl. Physiol., 24, 373-383.

4) Macklem P.T. \& Mead, J. (1967) Resistance of central and peripheral airways measured by retrograde catheter. J. appl. Physiol., 22, 395-401.

5) Macklem, P.T., Thurlbeck, W.M. \& Fraser, R.G. (1971) Chronic obstructive disease of small airways. Ann. intern. Med., 74, 167-177.

6) Nakamura, T., Takizawa, T., Takishima, T., Konno, K., Hoshino, F., Kurashina, T. \& Hatayama, T. (1969) Selective alveolo-bronchography in chronic pulmonary emphysema, Tohoku J. exp. Med., 99, 207-223.

7) Reid, L.M. (1955) Correlation of certain bronchographic abnormalities seen in chronic bronchitis with pathological changes. Thorax 10, 199-204.

8) Simon, G. \& Galbraith, H.J.B. (1953) Radiology of chronic bronchitis. Lancet, 2, $850-852$.

9) Weibel, E. (1963) Morphometry of the Human Lung, Springer, Berlin, Göttingen \& Heiderberg. 